\title{
Editorial
}

\section{Astrid Schreyögg}

Was im Milieu von Personalentwicklern früher oft als eher unbedeutende „Soft Skills“ bezeichnet wurde, erhält in den letzten Jahrzehnten einen zunehmend größeren Stellenwert. Wie kann sich nämlich im Zeitalter der Globalisierung eine Führungskraft, die nur über geringe Möglichkeiten von Einfühlungsfähigkeit oder Selbstreflexion verfügt, auf einem multinationalen Parkett bewegen? Oder wie können allzu „schlichte Gemüter“ die zunehmende Komplexität im Manageralltag heute noch bewältigen?Katz eröffnete schon 1974 die Debatte mit seinen „Schlüsselkompetenzen des Managements“, indem er eine Trias von technischen, konzeptionellen und sozialen Managementkompetenzen thematisierte. Unter „technischen Kompetenzen“ verstand er traditionelle Sachkompetenzen, die ein Ingenieur oder ein Betriebswirt schon in seinem Grundstudium erwirbt. Unter „konzeptionellen Kompetenzen“"verstand er aber eine bereits komplexere Fähigkeit, wie sich nämlich eine Führungskraft dauerhaft ihre Innovationsfähigkeit erhält, also nicht an den einmal entwickelten Erfolgsrezepten kleben bleibt, sondern immer wieder die ihr begegnenden Phänomene neu reflektiert und neu ausgestaltet. Als ,soziale Kompetenzen“" schließlich bezeichnete der Autor die Fähigkeit von Führungskräften, mit anderen Menschen effektiv und konstruktiv zusammenzuarbeiten, sich in sie einzufühlen und sie nicht nur als Funktionsträger, sondern auch als Menschen mit ihrem jeweiligen Sosein zu erfassen. Historisch wurde die Auseinandersetzung mit sozialen Kompetenzen besonders durch die Human Relations-Debatte in den USA der 1940er Jahre befeuert, denn nun wurde erstmalig deutlich, dass die Produktivität einer Firma keineswegs unabhängig ist von den sozialen Beziehungen, die sich innerhalb von Arbeitsgruppen entwickeln (Roethlisberger \& Dickson 1939). Solche Soft Skills erhalten heute in der Personalentwicklung einen fast selbstverständlichen Stellenwert. Dementsprechend werden sie auch durch Coaching und andere Maßnahmen der Personalentwicklung in vielen Firmen gezielt gefördert. Das vorliegende Heft von OSC fächert einige dieser Soft Skills auf und zeigt außerdem etliche Interventionen zu ihrer Förderung bei Fach- und Führungskräften.

Im ersten Hauptbeitrag geht Gabriela Heller anhand einer qualitativen Studie der Frage nach, ,inwieweit durch Business-Coaching auch eine allgemeine Entwicklung der

Online publiziert: 21.10 .2010

(C) VS Verlag für Sozialwissenschaften 2010

Dr. A. Schreyögg $(\bowtie)$

Breisgauer Str. 29,

14129 Berlin, Deutschland

E-Mail: info@schreyoegg.de 
Persönlichkeit verfolgt“" wird. Sie zeigt, dass in Coachingprozessen neben berufsbezogenen Sachthemen auch relevante Lebensthemen von Führungskräften bearbeitet werden sollten. Auf der Basis eines Rollenmodells von Lippmann zeigt die Autorin, dass eine nachhaltige Optimierung im Beruf letztlich auch der Persönlichkeitsentwicklung im Sinne von Life-Coaching bedarf. Und dabei ergibt sich dann automatisch die Förderung mehrerer Soft Skills. Im nachfolgenden Beitrag befasst sich Gerhard Liska mit dem „Zusammenhang von Führungsqualität und Burnout- bzw. Mobbingprävention“. Der Autor fragt, inwieweit solche destruktiven Phänomene als Erscheinungen des organisatorischen Systems zu werten sind, genauer als Ausdruck der Organisationskultur. Da diese aber in erster Linie von Führungskräften geprägt wird, ergibt sich hier immer die Frage nach deren sozialen Kompetenzen. Deshalb plädiert der Autor für eine gezielte prophylaktische Arbeit mit Führungskräften. Andreas Schulz zeigt, wie Supervision auf dem Hintergrund psychodramatischer Arbeit unterschiedliche „Selbst-Aspekte“ wie Selbstwert, Selbstachtung, Selbstsorge oder Selbstwirksamkeit befördern kann. Als Stärkung der ,inneren Autorität“ dient solche Arbeit nicht nur zur Stärkung der Identität, sondern auch zur Förderung der sozialen Kompetenz von Fach- und Führungskräften. Wolfram Schulze beschäftigt sich mit „Coaching im Rahmen von Bedrohungsmanagement“, wie es bei Stalking und besonders bei Amokläufen notwendig ist. Der Autor zeigt, dass Führungskräfte, die in ihrem Einflussbereich derartige Gewaltphänomene erleben, vor allem bei der Regulation von Emotionen unterstützt werden sollten. In solchen Fällen sei nämlich eine spezifische Facette sozialer Kompetenzen gefordert. Coaching mit diesem Fokus empfiehlt der Autor besonders männlichen Führungskräften, weil sie eher als weibliche dazu neigen, ihre Angst zu verleugnen und deshalb zu unangemessenen Handlungsstrategien verleitet werden. Rainer Bäcker konfrontiert uns im nächsten Beitrag sogar mit einer überaus unschönen Realität des modernen Managements. Im Zuge „humanistisch-psychologischer Weltverschönerung" sei den meisten Personalentwicklern entgangen, dass viele Führungskräfte heute regelrecht ,entgleist“ sind. Aus seiner Sicht als Managementdiagnostiker zeichnet er ein geradezu verheerendes Bild von manchen Führungskräften. Im Sinne von „Derailment“, was schon seit etlichen Jahren in der US-amerikanischen Forschung verhandelt wird, seien viele von ihnen eklatant überfordert bzw. der Geschwindigkeit und Komplexität des heutigen Wirtschaftslebens in keiner Weise gewachsen. Der Autor gibt zu bedenken, dass solche Phänomene mit traditionellen Formen der Managementdiagnostik kaum zu erfassen sind, er plädiert vielmehr für Auswahlverfahren, bei denen viel umfassender auf soziale Faktoren, also die Soft Skills geachtet wird.

In ihrem Praxisbericht zeigt uns Nadja Lehmann, dass in der Supervision immer mal wieder biographische Exkurse notwendig sind. In ihrem Fallbeispiel geht es um eine analoge Erfahrung von Bürgerkriegsflüchtlingen und Menschen aus der DDR nach der Wende. Diese heute allzu häufig verdrängten Kränkungen, die Menschen in der Nachwendezeit durch Westdeutsche erlebt haben, hinterließen besonders bei der jungen Generation ihre Spuren.

Im Diskurs stellt Ingo Steinke drei Modelle für das Qualitätsmanagement vor, die mit ihren jeweiligen Besonderheiten und Vorgehensweisen beschrieben und im Hinblick auf eine Anwendbarkeit für das Coaching bewertet werden. Angesichts dieses Beitrags wird deutlich, dass es sich hier um eine längst überfällige Debatte handelt. 